\title{
La Gestion Pedagogique Des Medersas Au Maroc, Entre Tradition Et Reforme
}

\section{Lahoucine Aguinou, Docteur en Sciences de l'Éducation}

Spécialité : Enseignement et Formation

Groupe de recherche et d'analyse du système d'enseignement et de formation

(GRAFE), Faculté des Sciences de l'Éducation, Université Mohamed V,

Rabat, Maroc

Doi:10.19044/esj.2019.v15n13p374 URL:http://dx.doi.org/10.19044/esj.2019.v15n13p374

\section{Résumé}

Cet article, qui s'inscrit dans le cadre des Sciences de l'éducation, tente d'étudier la gestion et le fonctionnement pédagogique des médersas au Maroc. Il met en évidence les modifications apportées par la réforme de l'an 2002 au niveau du fonctionnement pédagogique de ces écoles, qui sont des acteurs du système d'enseignement traditionnel. Pour réaliser ce travail, nous adoptons une méthode qualitative et descriptive. Notre approche est pluridisciplinaire. Nous mobilisons une recherche documentaire, qui se base principalement sur les textes de loi régissant la mise en œuvre de la réforme, les résultats de notre thèse, les ouvrages et les articles qui traitent notre problématique.

L'analyse des résultats de cette étude montre que la réforme a modifié la gestion et le fonctionnement pédagogique des écoles traditionnelles réformées. Quant à ceux des médersas non réformées, ils n'ont pas connu de grands changements.

Mots clés: médersa, réforme, gestion pédagogique, fonctionnement pédagogique. 


\title{
The Pedagogical Management of Madrassas in Morocco, Between Tradition and Reform
}

\section{Lahoucine Aguinou, Docteur en Sciences de l'Éducation \\ Spécialité : Enseignement et Formation}

Groupe de recherche et d'analyse du système d'enseignement et de formation

(GRAFE), Faculté des Sciences de l'Éducation, Université Mohamed V,

Rabat, Maroc

\begin{abstract}
This article, part of the educational sciences, attempts to study the management and pedagogical functioning of madrassas in Morocco. It highlights the changes made by the reform of the year 2002 in the educational functioning of these schools, which are actors in the traditional education system. To carry out this work, we adopt a qualitative and descriptive method. Our approach is multidisciplinary. We mobilize a literature search, which is based mainly on legislation governing the implementation of the reform, the results of our thesis, the books and articles that deal with our problems.

The analysis of the results of this study shows that the reform has changed the management and pedagogical functioning of the traditional schools reformed. As for those unreformed madrassas, they have not known great changes.
\end{abstract}

Keywords: medarsa, reform, pedagogical management, pedagogical functioning.

\section{Introduction}

$\mathrm{Au}$ Maroc, le système d'enseignement traditionnel, appelé aussi « Nizam al-Taà 'lim al-À'atiq », se compose de cinq institutions, à savoir : la mosquée, la zaouïa, l'école coranique (ou $\mathrm{msid}$ ), l'école traditionnelle (appelée aussi: école des sciences religieuses, madrassa, médersa et medarsa) et la jamià 'a (Université d'Al-Qaraouiyin, située à la ville Fès).

Les Médersas ${ }^{26}$, qui nous intéressent dans le cadre de cette étude, constituent la phase secondaire dudit système. Elles reçoivent les sortants des écoles coraniques, qui ont réussi à mémoriser le Coran. Jadis, ces établissements étaient gérés d'une manière autonome. C'étaient les maitres,

${ }^{26}$ Pendant l'année scoalire 2017/2018, le nombre de ces établissements au Maroc était 286. Ils ont reçu environ 35004 élèves. Repéré le 21 mars 2019 sur: http://www.habous.gov.ma/الاحصاء_الددرسي.html 
$\left(\right.$ faqih $^{27}$ ou $\left.t a l b^{28}\right)$ qui assuraient leur gestion, qui était la même, dans tout le Royaume. Après l'indépendance du pays, ces écoles ont vécu des décennies de crise. Elles étaient marginalisées par l'État. L'enseignement qu'elles dispensaient ne permettait pas d'intégrer les métiers modernes. C'est à partir de l'an 2002, que leur fonctionnement a connu des modifications importantes, apportées par la réforme, qui s'inscrit dans le cadre de la restructuration du champ religieux. La mise en œuvre de la nouvelle politique a réduit l'autonomie de ces écoles. Elles sont désormais soumises au contrôle de l'État.

\section{Problématique}

Jadis, la gestion pédagogique de la médersa était assurée par ses maitres, qui n'étaient soumis à aucun contrôle. Ils enseignaient les contenus de leurs choix. Le fonctionnement pédagogique de l'école ne dépendait que des compétences personnelles des apprenants (talabas). Depuis l'an 2002, suite à la mise en œuvre de la réforme du secteur d'enseignement traditionnel, leur autonomie de gestion pédagogique s'est réduite. Elle est soumise, désormais, au contrôle du Ministère des Habous et des Affaires Islamiques (Aguinou, 2018, p.210). Le changement a touché toutes les structures (organisationnelles et physiques), la gestion (administrative, pédagogique, financière), les équipements, certains rôles et pouvoirs des écoles traditionnelles. Dans cet article, nous nous limitons à l'étude de quelques modifications apportées au fonctionnement pédagogique desdites écoles. Nous tentons de répondre à la question principale suivante:

- Quelles sont les changements apportés par la réforme de l'an 2002 au fonctionnement pédagogique des médersas, notamment au niveau des contenus, de la gestion du temps, des traditions d'apprentissage, des méthodes d'enseignement, d'évaluation, des congés et des diplômes?

\section{Hypothèses}

Nos deux hypothèses, présentées ci-après, présentent des propositions de réponses à la question posée :

- Les madrassas non réformées conservent leurs modes de gestion et de fonctionnement pédagogiques ancestraux ;

- La réforme de l'an 2002 a apporté des modifications importantes aux modes de gestion et de fonctionnement pédagogiques des médersas réformées dans les domaines précités dans la problématique.

${ }^{27}$ Faqih est un mot arabe dont le pluriel est: Foqahas. Il désigne le maitre chargé d'enseigner le â'ilm (le savoir) dans les écoles traditionnelles.

${ }^{28}$ Le mot $t a l b$ est employé ici pour désigner « l'enseignant du Coran » à l'école traditionnelle. 


\section{Objectifs}

Il est important de signaler que cette étude n'a pas pour objet d'évaluer la réforme de l'enseignement traditionnel au Maroc. Elle a pour objectif ultime de contribuer à la construction de futures recherches dans le système d'enseignement traditionnel au Royaume. Elle tente d'analyser la relation de la réforme avec le fonctionnement pédagogique des madrassas, à travers l'étude des modifications repérées sur le fonctionnement des domaines précités dans la problématique.

\section{Méthodologie}

Notre recherche, qui s'inscrit dans le cadre des Sciences de l'Éducation, est qualitative. Elle adopte une méthode descriptive, qui dépeint le système d'enseignement dispensé par les écoles traditionnelles marocaines. Elle produit et analyse des données descriptives relatives aux structures dudit enseignement, en insistant sur l'étude du fonctionnement pédagogique desdites écoles.

Notre approche est interdisciplinaire. Elle nous permet de prendre en compte la totalité des données relatives à la problématique.

Pour réaliser ce travail, nous mobilisons une recherche documentaire, qui se base essentiellement sur la loi 13.01, régissant ledit enseignement et ses textes d'application. Nos recourons également à nos propres études réalisées dans le cadre de la préparation de notre thèse dans le domaine des Sciences de l'Éducation, soutenue au mois de novembre 2018, intitulée : « la Madrassa au Maroc, entre tradition et modernité. Cas de la madrassa de Sidi Ouaggag, CT d'Aglou, province de Tiznit (2012-2017) ». Quelques ouvrages et articles, qui ont traité l'objet de ce travail, sont également consultés.

Avant de traiter notre problématique, Il convient de présenter quelques éclairages quant aux types des madrassas, aux cycles d'enseignement traditionnel et leurs durées.

\section{Les types des médersas}

Les médersas sont souvent indépendantes des mosquées et annexées aux zaouïas. Elles reçoivent les élèves venant des écoles coraniques (msids) créées dans tous les douars et quartiers du pays. Elles sont des biens habous, placées sous la tutelle du Ministère des Habous et des Affaires Islamiques, même si elles sont construites par les habitants. L'enseignement dispensé par ces institutions se caractérise par la mémorisation du Coran, la diffusion du savoir religieux, la bienséance, la solennité et la fidélité de ses acteurs aux principes de la nation ${ }^{29}$. Avant la réforme du secteur en l'an 2002, ces établissements se

${ }^{29}$ Source: le discours prononcé par le ministre des Habous et des Affaires Islamiques, lors de l'inauguration de la deuxième session du moussem annuel des écoles traditionnelles, intitulé : « Grand commandement [en arabe] », organisé à Taroudant du 18 au 25 mars 2014, par la 
subdivisaient en deux types: les écoles des sciences religieuses et les écoles du Coran (Al-Ilghi, 1998, p.110). Aujourd'hui, Ils se subdivisent en trois types, à savoir: les écoles réformées (publiques et privées), les madrassas non réformées (elles refusent le changement et préservent leurs modes de fonctionnement traditionnels et ancestraux), et enfin les écoles traditionnelles hybrides (elles dispensent, à la fois, l'enseignement séculaire et l'enseignement modernisé).

Quant aux élèves de cet enseignement, ils se répartissent en trois catégories, à savoir :

- Al-Coraniyouns, qui forment le premier groupe, s'intéressent à la mémorisation du Coran, sous la supervision du talb.

- les talabas du â'ilm (du savoir), qui ont déjà mémorisé une grande partie du Coran, constituent la deuxième catégorie. Ils se consacrent à l'étude des sciences religieuses.

- les élèves de l'enseignement réformé, forment la dernière catégorie.

\section{Les cycles d'enseignement traditionnel et leurs durées}

Avant la réforme, les talabas pouvaient rester à la madrassa tant qu'ils désiraient, même si la tradition fixe la durée maximale des études, dans l'école, à dix ans (AGUINOU, 2018, p.190).

Depuis l'an 2002, l'enseignement traditionnel réformé comprend les cycles suivants: préscolaire, primaire, secondaire collégial, secondaire qualifiant et terminal (Art. 2, Dahir 1-02-09 du 29 janvier 2002). La durée de chaque cycle est, désormais, équivalente à celle appliquée dans le cycle correspondant du système d'enseignement national moderne (Art. 3, Dahir 102-09). C'est ainsi que les études durent : deux ans au préscolaire, six ans au primaire, et trois ans dans chacun des trois cycles suivants: collégial, secondaire, et terminal. La poursuite des études supérieures aux cycles master et doctorat est désormais possible.

\section{Les programmes de l'enseignement traditionnel}

Nous apportons, ici, des éclairages quant aux contenus enseignés dans les madrassas non réformées et ceux enseignés dans les écoles traditionnelles réformées.

\section{Dans les médersas non réformées}

Les études consistent, principalement, à apprendre par cœur le Coran, des cours de langue arabe, de syntaxe et des fondements essentiels de la religion de l'Islam. Les maitres enseignent, en plus des lectures du Coran,

fondation Souss des écoles traditionnelles. Repéré le 11 février 2019 sur: https://www.maghress.com/attajdid/110898. 
notamment la lecture de Warch, le Hadith, la Sira al-Nabawiya, l'exégèse, le droit musulman, les fondements du fiqh, la grammaire, la conjugaison, la métrique, la rhétorique, le calcul, la logique, les sciences occultes, la littérature arabe et l'astronomie.

\section{Dans les médersas réformées}

La réforme introduit un enseignement trilingue (Arabe, Français et Anglais). En l'an 2011, les contenus imposés par la réforme ont été révisés, sous la supervision des commissions pédagogiques. Il est désormais interdit d'enseigner les programmes non autorisés. Les nouveaux contenus comportent, dans la proportion des deux tiers de leur volume horaire, des matières obligatoires enseignées dans les établissements de l'enseignement public y compris les cours des langues, des mathématiques et de l'éducation physique (Art. 4, Dahir 1-02-09). Le tableau suivant détaille les nombres des matières enseignées, des heures par semaine et par an, pour chaque niveau d'enseignement dans les médersas réformées.

\begin{tabular}{|c|c|c|c|c|}
\hline Cycle & $\begin{array}{c}\text { Nombre de } \\
\text { matières }\end{array}$ & Niveau & $\begin{array}{c}\text { Nombre } \\
\text { d'heures/semaine }\end{array}$ & $\begin{array}{c}\text { Nombre } \\
\text { d'heure/an }\end{array}$ \\
\hline \multirow{6}{*}{ Primaire } & \multirow{6}{*}{17} & 1 & 33 & 1122 \\
\hline & & 2 & 34 & 1156 \\
\hline & & 3 & 35 & 1180 \\
\hline & & 4 & 35 & 1180 \\
\hline & & 5 & 35 & 1180 \\
\hline & & 6 & 35 & 1180 \\
\hline \multirow{3}{*}{ Collégial } & \multirow{3}{*}{20} & 1 & 35 & 1180 \\
\hline & & 2 & 35 & 1180 \\
\hline & & 3 & 35 & 1180 \\
\hline \multirow{3}{*}{ Secondaire } & \multirow{3}{*}{19} & 1 & 34 & 1156 \\
\hline & & 2 & 34 & 1156 \\
\hline & & 3 & 34 & 1156 \\
\hline
\end{tabular}

Source: www.habous.gov.ma

\section{La gestion du temps dans les médersas}

Afin de bien appréhender les changements apportés par la nouvelle politique sur la gestion du temps dans les madrassas au Maroc, nous présentons, en premier lieu, le déroulement d'une journée d'étude dans les médersas non réformées. En second lieu, nous mettons en lumière cette gestion dans les écoles réformées.

\section{Dans les écoles non réformées}

Le temps est géré suivant des traditions ancestrales. Les séances de la matinée commencent au lever du soleil et continuent jusqu'à midi. Les cours reprennent juste après la prière d'al-Dohr (de midi), et s'achèvent après la prière d'al-ÂA'asr (de l'après-midi). Au coucher du soleil, les talabas 
participent à la lecture collective du Coran, et à la prière d'al-Maghreb (du coucher de soleil) dans la mosquée. Ils retournent, ensuite, à l'école pour y rester jusqu'à l'heure de la prière d'al-Â'icha (de la nuit). Certains élèves continuent à apprendre par cœur le livre sacré, ou à réviser leurs cours jusqu'à minuit (Aguinou, 2018, p. 213).

\section{Dans les écoles réformées}

Aujourd'hui, la gestion du temps dans ces écoles se complique. Leur fonctionnement actuel comporte quelques actions sous la supervision du faqih. Certaines d'autres sont sous la direction de l'administration de l'école, et d'autres sous la responsabilité de l'association de l'établissement et la communauté. Leurs activités (formelles ou informelles) mobilisent tous ces responsables, à titre obligatoire ou facultatif. Leur engagement pose des problèmes quant à leurs responsabilités juridiques et à leur motivation. D'un côté, le chart (le contrat économique liant le maitre et les habitants) ne reconnait pas le désengagement du faqih et du talb de leurs responsabilités, hors les nouvelles vacances annuelles. Ils sont tenus d'assister aux lectures collectives quotidiennes du Coran et de diriger les prières, même pendant les congés hebdomadaires (jeudis et vendredis). Quant aux staffs administratif et pédagogique, leur participation aux diverses activités, hors temps de service, n'est que volontaire (Aguinou, 2018, p. 213).

Le nouveau programme est fixé par le ministère de tutelle. La présentation des cours et l'évaluation des apprenants sont assurées par des enseignants, suivant des méthodes pédagogiques et des outils d'apprentissage modernes.

La gestion du temps dans ces écoles est bien règlementée. Les emplois du temps sont préparés par le surveillant général et validés par le directeur de l'école. La présence des élèves à la mosquée, pendant les heures des cinq prières et celles des deux lectures collectives du Coran, est obligatoire.

Les talabas du â'ilm, quant à eux, en plus de l'apprentissage des principes de la langue arabe, les moutouns (recueils), les principes du fiqh, etc, ils participent auxdites lectures collectives et aux cinq prières quotidiennes. Après la prière d'al-Dohr, ils se réunissent autour du faqih, qui présente les cours de son choix. Ils révisent leurs planchettes avant de dormir. Ils ont le droit de demander des séances de soutien à leur maitre, pendant la nuit, après avoir assisté à la prière d'al-Â'icha (Aguinou, 2018, p. 228).

\section{Les traditions d'apprentissage dans les médersas}

Dans toutes les écoles traditionnelles non réformées du Royaume, ces traditions se ressemblent, suivant les domaines de spécialisation. Dans les madrassas du Coran, l'enseignant répartit les apprenants en petits groupes, selon les niveaux. Les plus avancés aident les nouveaux arrivants. L'apprentissage et la mémorisation s'effectuent à travers trois phases 
différentes. La première séance se consacre à l'écriture et la récitation, la deuxième à la révision et la récitation, et la dernière à la consolidation et la composition (Oubella, 1995, p.66). La première commence juste après l'aube et s'achève à la matinée. Les talabas révisent, pour une dernière fois, avant de réciter ce qu'ils ont mémorisé, devant leur enseignant, qui corrige les fautes au fur et à mesure des récitations. Enfin, ils effacent leurs planchettes, et les exposent au soleil pour les faire sécher. La deuxième séance s'étale sur trois étapes. La première commence avant l'aube, la deuxième pendant les séances de la matinée, et la dernière après la prière d'al-Dohr. Pendant la séance de consolidation et de composition, les talabas récitent ce qu'ils ont appris récemment. La récitation se fait collectivement avec la participation du talb enseignant, qui corrige les fautes de lecture. Cette activité se présente, entre les prières d'al-Maghreb et d'al-Â'icha et aussi pendant la période de révision d'avant l'aube, notamment par les élèves brillants (Oubella, 1995, p.67).

Après la réforme du secteur, les foqahas et les tolbas conservent les traditions ancestrales d'enseignement du Coran et des sciences religieuses. Les pratiques pédagogiques, dans les écoles réformées, quant à elles, sont inspirées des pratiques adoptées dans le système scolaire moderne.

\section{Les méthodes d'enseignement traditionnel}

Les méthodes suivies par les maitres pour enseigner le Coran et les programmes anciens, n'ont pas subi de grands changements, depuis des siècles. Elles se font, selon les traditions ancestrales. Elles partent de la partie vers le tout, et de la découverte au détail ( Erckmann, 1885, p.92). Elles se basent sur la transmission du savoir et se pratiquent suivant un style individuel. La mémorisation s'effectue en fonction du rythme et des capacités de mémorisation propre à chaque apprenant. Le maitre appelle ses talabas à tour de rôle pour lire ou réciter les teneurs de leurs plaques de bois. Les anciens élèves aident les nouveaux arrivants à mémoriser leurs cours. Après la réforme, les nouveaux enseignants (lauréats des universités modernes) sont formés pour enseigner les matières laïques, imposées par la réforme, suivant les méthodes du système moderne. Ils sont soumis au contrôle de l'autorité de tutelle. Le non-respect des règlementations en vigueur est sanctionné.

\section{L'évaluation des talabas}

Jadis, dans toutes les écoles du Royaume, l'évaluation des élèves s'effectuait oralement par le maitre, chaque jour, à travers la mémorisation des planchettes. Elle n'était soumise à aucun contrôle administratif.

Aujourd'hui, dans les écoles réformées, les nouveaux enseignants, évaluent les niveaux des élèves suivant des méthodes imposées par la réforme. Les modalités d'évaluation et le régime des examens, les coefficients afférents à chaque matière, selon le cycle d'enseignement, sont régies par la loi (Art. 2, 
Dahir 1-02-09). L'organisation de ces actions s'effectue suivant les orientations ministérielles (Arrêté ministériel 873.06 du 03 mai 2006). Le nonrespect de ces dernières est sanctionné (Art. 22, Dahir 1-02-09). Dans les écoles non réformées, les maitres continuent à évaluer, oralement, leurs talabas suivant les modalités antiques.

\section{Les congés de l'enseignement traditionnel}

Les congés hebdomadaires : Dans toutes les écoles, les cours s'arrêtent tous les jeudis et vendredis ${ }^{30}$.

Les congés annuels : Ce sont les vacances des fêtes religieuses suivantes :

- aïd al-Fitre : du quinzième jour du mois de Ramadan au huitième jour du mois de chowal ;

- aïd al-Adha : du premier jour du mois de dou al-Hija au lendemain d'aïd alKabir, c'est-à-dire au dix-huitième jour du même mois ;

- aïd al-Mawlid al-Nabaoui: du premier jour du mois de rabi'â-I au lendemain du jour d'al-Mouloud, le vingtième jour du même mois ;

- les dixième et onzième jours du mois de moharrem, les cours s'arrêtent dans les établissements de l'enseignement traditionnel pour célébrer la fête d' $\hat{A}$ achoura.

Les congés occasionnels : Ils ne dépassent pas une seule journée de repos. Ce sont les jours suivants:

- le jour où le talb reçoit un invité ;

- le jour d'une fête célébrée dans le douar (le jour du mariage par exemple) ;

- le jour de la fête organisée par l'un des talabas à l'occasion de sa khatma ${ }^{31}$, appelé aussi : le jour de souna (AL-Ilghi, 1998, p.100).

- le jour de zerda: quand une personne bienfaisante apporte de la viande et des légumes à l'école, les élèves se chargent de fêter l'occasion et inviter à manger des personnes ayant mémorisé le Coran.

Pendant tous ces congés, les talabas partent chaque jour aux madrassas ou aux mosquées les plus proches, pour maintenir leur lien avec le Coran et réciter ce qu'ils ont déjà appris, pour ne pas l'oublier.

Les élèves internes, qui ne partent pas chez eux, peuvent rester à l'école pendant ces vacances.

\section{Les diplômes de l'enseignement traditionnel}

Jadis, l'attestation d'al-Ijaza al-Â'ilmiya, délivrée par le maitre (considéré comme : cheikh), assurait la reconnaissance de l'instruction et le savoir du sortant de la madrassa (Al-Mokhtar Soussi, 1960, p.68). Cette tradition existait en Andalousie, depuis l'époque des moravides (Hmitou, 2006, p. 850). Elle

${ }^{30}$ C'était Omar Bnou Al-Khattab, le compagnon du Prophète Mohamad, qui a décidé que les jeudis et les vendredis soient les jours de congé des élèves des écoles coraniques.

${ }^{31}$ Chaque achèvement de la mémorisation des soixante hizbs du Coran est appelé: khatma. 
représentait l'autorisation (orale ou écrite) du cheikh à son disciple (taleb) de diffuser son savoir (Aguinou, 2018, p. 225). Elle lui permettait d'intégrer le métier d'enseignant. Après la mise en œuvre de la réforme, les élèves de l'enseignement traditionnel réformé, et ceux de l'enseignement moderne obtiennent les mêmes certificats scolaires, attestant de leurs niveaux d'études.

Ces certificats sont (Note ministérielle 100 du 31 mai 2013):

- le certificat d'enseignement primaire traditionnel;

- le certificat d'enseignement collégial traditionnel;

- le certificat du baccalauréat de l'enseignement secondaire traditionnel.

Les médersas réformées, sont désormais liées à l'enseignement moderne (public ou privé) par des passerelles. Ces dernières permettent aux élèves de l'École moderne de rejoindre le système d'enseignement traditionnel et aux talabas de ce dernier de rejoindre les classes du système moderne ou les cycles de la formation professionnelle.

\section{Conclusion}

Dans le cadre de la restructuration du champ religieux marocain, le système d'enseignement traditionnel, dont les madrassas sont actrices, a été réformé en l'an 2002. La modernisation des modes de gestion et de fonctionnement de ces écoles traditionnelles influence inévitablement leur structure et leur autonomie de gestion. Cette situation nous a incité, dans le cadre de ce travail, à interroger le fonctionnement pédagogique de ces établissements, après la mise en oeuvre de la nouvelle politique.

À l'issue du traitement et d'analyse des données collectées lors de notre recherche documentaire, les deux hypothèses de cette recherche sont confirmées.

La gestion pédagogique est la même dans toutes les madrassas non réformées du Royaume. Elle est assurée par les maitres (faqih ou talb), qui enseignent des contenus de leurs choix, suivant des méthodes anciennes. Les études commencent du matin jusqu'à minuit, dans certaines écoles. Les élèves bénéficient de quelques pauses de repos, suivant leurs niveaux et leurs domaines de spécialisation. Leur évaluation s'effectue oralement par le maitre. Les études s'arrêtent tous les jeudis et vendredis, et pendant les fêtes religieuses et occasionnelles. Les formations dispensées ne sont pas diplomantes.

Quant aux madrassas réformées, leur autonomie de gestion s'est réduite. Ces établissements sont soumis, désormais, au contrôle de l'État. Le directeur de l'école est devenu le responsable et le représentant officiel de l'établissement. Les nouveaux programmes unifiés, introduits, sont enseignés, par des lauréats des universités modernes, suivant des méthodes et des pratiques inspirées du système moderne. Les emplois du temps sont validés par l'administration. Des durées des études sont fixées pour chaque cycle 
d'enseignement. Des examens sont organisés et les formations dispensées sont désormais diplomantes. Elles permettent l'accès aux métiers modernes. En plus des congés du système traditionnel, les élèves de ces écoles bénéficient des congés du système moderne.

\section{References:}

1. AGUINOU, L. (2018). La madrassa au Maroc: entre tradition et modernité. Cas de la madrassa de Sidi Ouaggag, CT d'Aglou, province de Tiznit (2012-2017). Thèse, Faculté des Sciences de l'Éducation, Université Mohamed V, Rabat, Maroc.

2. AL-ILGHI, S. (1998). La première école: Description générale de l'enseignement préscolaire dans l'école coranique à Souss - Cas de l'école d'Ilgh-[en arabe]. Casablanca : Imprimerie Al-Najah.

3. AL-MOKHTAR SOUSSI, M. (1960). Al-Ma'soul, 19 [en arabe]. Casablanca: Imprimerie Al-Jamiâ'a.

4. Arrêté du ministre des Habous et des Affaires Islamiques n ${ }^{\circ} 873.06 \mathrm{du}$ 03 mai 2006, ayant trait à l'organisation du système d'enseignement traditionnel (B.O 5449 du 21 Aout 2006).

5. Dahir $\mathrm{n}^{\circ}$ 1-02-09 du 15 kaada 1422 (29 janvier 2002) portant promulgation de la loi $\mathrm{n}^{\circ} 13-01$ relative à l'enseignement traditionnel (B.O n 4980 du 21/02/2002).

6. ERCKMANN, J. (1885). Le Maroc moderne. Paris: Librairie Coloniale. Récupéré en janvier 2019 sur gallica.bnf.fr

7. HMITOU, A. (2006). La vie du Kottab et littérature de la Mahdara [en arabe]. Rabat: Éditions Abou Ragrague.

8. Note ministérielle $\mathrm{n}^{\circ} 100$ du 31 mai 2013 , relative à délivrance des attestations de réussite en primaire, repérée sur: http://www.habous.gov.ma/files/التعليم/20\%//almodakkirat_al_am ma_enseignement_traditionnel_2013.pdf

9. OUBELLA, B. (1995), Le mécanisme de l'école traditionnelle entre la réalité et l'autonomie. Dans l'association Adouz pour le développement et la coopération, les écoles scientifiques traditionnelles - perspectives de réforme et intégration dans l'environnement contemporain [en arabe], 55-68. 